Research Article

\title{
On the Positive Operator Solutions to an Operator Equation $X-A^{*} X^{-t} A=Q$
}

\author{
Kaifan Yang \\ School of Mathematics and Computer Science, Shaanxi University of Technology, Hanzhong, Shaanxi, China \\ Correspondence should be addressed to Kaifan Yang; 82509496@qq.com
}

Received 11 July 2021; Accepted 11 September 2021; Published 30 September 2021

Academic Editor: Jie Wu

Copyright (C) 2021 Kaifan Yang. This is an open access article distributed under the Creative Commons Attribution License, which permits unrestricted use, distribution, and reproduction in any medium, provided the original work is properly cited.

In this paper, the positive operator solutions to operator equation $X-A^{*} X^{-t} A=Q(t>1)$ are studied in infinite dimensional Hilbert space. Firstly, the range of norm and the spectral radius of the solution to the equation are given. Secondly, by constructing effective iterative sequence, it gives some conditions for the existence of positive operator solutions to operator equation $X-$ $A^{*} X^{-t} A=Q(t>1)$. The relations of these operators in the operator equation are given.

\section{Introduction}

Operator algebra has played an important role in the subject of functional analysis; it has been considered by many authors. At the same time, operator equation is one of the hottest topics in operator theory. The researches on the positive solutions to operator equations began in 1990s; it has been applied to many fields such as dynamic programming [1], stochastic filtering, control theory $[2,3]$, and statistics [4]. In recent years, operator equation attained a great development and many scholars put into studying different kinds of operator equations (see [5-11]).

In this paper, let $H$ be infinite dimensional Hilbert space and $\mathrm{B}(\mathrm{H})$ denote the set of all bounded linear operators on $H$; we will consider nonlinear operator equation.

$$
X-A^{*} X^{-t} A=Q \text {, }
$$

in $H$; here, $A, Q \in B(H)$ with $Q>0, A^{*}$ is the adjoint of $A$.

In the past few years, many authors used different iterative methods for computing the positive definite solutions to equation (1) in finite dimensional space. In this paper, we extend the study of operator equation (1) from finite dimensional space to infinite dimensional Hilbert space. Some necessary conditions for the existence of positive operator solutions to operator equation (1) are derived. Furthermore, conditions under which operator equation (1) has positive operator solutions are obtained.
For $A \in B(H), A^{*}, \sigma(A),\|A\|$ denote the adjoint, the spectrum, and the norm of $A$, respectively. If $A x, x \geq 0$ for all $x \in H$, then $A$ is said to be a positive operator and denoted by $A \geq 0$. For positive operators in $B(H)$, the following conclusions are obvious:

(1) For $P \geq Q>0$, we have $P^{-1} \leq Q^{-1}$.

(2) For positive operator $P, \lambda_{\min }(P) I \leq P \leq \lambda_{\max }(P) I$, where

$$
\begin{aligned}
& \lambda_{\text {min }}(P)=\min \{\lambda: \lambda \in \sigma(P), P>0\}, \\
& \lambda_{\text {max }}(P)=\max \{\lambda: \lambda \in \sigma(P), P>0\} .
\end{aligned}
$$

\section{Main Results and Proofs}

Lemma 1 (see [12]). Let $A, B \in B(H)$. If $A \geq B$, then $\|A\| \geq\|B\|$.

Lemma 2 (see [12]). Let $A$ and $B$ be self-adjoint operators in $B(H)$. If $A \leq B$, then for any $T \in B(H)$, we have $T^{*} A T \leq T^{*} B T$.

Proposition 1 (see [12]). If $A \in B(H)$ is normal, then the $C^{*}$-algebra generated by $A$ is commutative. 
Theorem 1. If the operator equation (1) has a positive operator solution $X$, then

$$
\|Q\| \leq\|X\| \leq\|Q\|\left(1+\left\|Q^{(-t / 2)} A Q^{(-1 / 2)}\right\|\right)^{2} .
$$

Proof. If the operator equation (1) has a positive operator solution $X$, from $A^{*} X^{-t} A>0$, we can obtain $X \geq Q$. From Lemma 2 , it is easy to see $\|X\| \geq\|Q\|$. From equation (1),

$$
\begin{aligned}
X & =Q+A^{*} X^{-t} A \leq Q+A^{*} Q^{-t} A \\
& =Q^{(1 / 2)}\left(I+Q^{-(1 / 2)} A^{*} Q^{-t} A Q^{-(1 / 2)}\right) Q^{(1 / 2)} .
\end{aligned}
$$

We can obtain $\|X\| \leq\|Q\|\left(\left\|I+Q^{-(1 / 2)} A^{*} Q^{-t} A Q^{-(1 / 2)}\right\|\right)=$ $\|Q\|\left(1+\left\|Q^{-(t / 2)} A Q^{-(t / 2)}\right\|^{2}\right)$. That is, $\|Q\| \leq\|X\| \leq$ $\|Q\|\left(1+\left\|Q^{(-t / 2)} A Q^{(-1 / 2)}\right\|^{2}\right)$. The theorem is proved.

Theorem 2. If $A$ is invertible, then equation (1) has a positive operator solution.

Proof. Let $\phi(X)=Q+A^{*} X^{-t} A$, then $\phi$ is continuous for any $X \in\left[Q, Q+A^{*} Q^{-t} A\right]$. Clearly, $\phi(X) \geq Q$ for any $X$, combination with the proof of Theorem 1, we have
$\phi(X)=Q+A^{*} X^{-t} A \leq Q+A^{*} Q^{-t} A, \quad$ that is, $\phi(X) \in$ $\left[Q, Q+A^{*} Q^{-t} A\right]$; this implies $\phi$ is a mapping to itself on $\left[Q, Q+A^{*} Q^{-t} A\right]$. By the fixed point theorem, $\phi$ has a fixed point $X_{0}$ in $\left[Q, Q+A^{*} Q^{-t} A\right]$ such that $\phi\left(X_{0}\right)=X_{0}$, i.e., $X_{0}=Q+A^{*} X_{0}{ }^{-t} A$, that is, $X_{0}$ is a positive operator solution to equation (1).

Theorem 3. Let $A \in B(H)$. The operator equation $X-A^{*} X^{-t} A=Q$ has a positive invertible operator solution $X$ if and only if $A$ has the factor decomposition $A=\left(W^{*} W\right)^{(t / 2)} B$, where $W, B$ satisfy $W^{*} W-$ $B^{*} B=Q$ and $W$ is invertible.

Proof. If operator equation (1) has a positive operator solution $X$, let $W=\sqrt{X}$, then $W^{*}=W$ and $W$ is invertible, so $X=W^{*} W$. According to equation (1), we have

$$
W^{*} W-\left(\left(W^{*} W\right)^{-(t / 2)} A\right)^{*}\left(W^{*} W\right)^{-(t / 2)} A=Q \text {. }
$$

Let $\left(W^{*} W\right)^{-(t / 2)} A=B$, then $A=\left(W^{*} W\right)^{(t / 2)} B$. From equality (5), we obtain $W^{*} W-B^{*} B=Q$. Conversely, if $A$ has the factor decomposition $A=\left(W^{*} W\right)^{(t / 2)} B, W, B$ satisfy $W^{*} W-B^{*} B=Q$ and $W$ is invertible. Let $X=W^{*} W$, then

$$
X-A^{*} X^{-t} A=W^{*} W-\left(\left(W^{*} W\right)^{(t / 2)} B\right)^{*}\left(W^{*} W\right)^{-t}\left(W^{*} W\right)^{(t / 2)} B=W^{*} W-B^{*} B=Q
$$

That is, $X$ is a positive operator solution to equation (1).

In [6], it proves that if $\mathrm{A}$ is not invertible and then $X$ is a positive solution to $X+A^{*} X^{-2} A=I$, then $\lambda_{\max }(X)=1$. In finite dimensional space, if $A$ is not invertible, then $N(A) \neq 0$, but in infinite dimensional space, if A is not invertible, $N(A)$ maybe a null space; the lemma in [6] is not held in infinite dimensional space, but the following conclusion holds.

Theorem 4. If $X-A^{*} X^{-t} A=Q$ has positive operator solution $X$, then $\|X\|=\|Q\|$ if and only if $A$ is not bounded below.

Proof. From Theorem 1, we know $\|X\| \geq\|Q\|$ for any positive operator solution to equation (1).

Necessary. If $X-A^{*} X^{-t} A=Q$ has positive operator solution $X$ and $\|X\|=\|Q\|$, then $\omega(X)=\gamma(X)=\|X\|=\|Q\|$, hence there exists unit sequence $\left\{x_{n}\right\}_{n=1}^{+\infty}$ such that $\left(X^{s} x_{n}\right.$, $\left.x_{n}\right) \longrightarrow b(n \longrightarrow \infty)$. For any unit vector $x \in H$, we have

$$
\langle X x, x)\rangle=\left\langle\left(A^{*} X^{-t} A+Q\right) x, x\right\rangle=\langle Q x, x\rangle+\left\langle A^{*} X^{-t} A x, x\right\rangle,
$$

hence $\left(X^{-t} A x_{n}, A x_{n}\right) \longrightarrow 0$. On the other hand, $\left(X^{-t} A x_{n}, A x_{n}\right)=\left\|X^{-(t / 2)} A x_{n}\right\|^{2} \geq\left(\left\|A x_{n}\right\|^{2} /\left\|X^{(t / 2)}\right\|^{2}\right)$.

Hence, $A x_{n} \longrightarrow 0$, therefore $A$ is not bounded below.

Sufficient. Assume $\|X\|>\|Q\|$ for any positive operator solution $X$, then $X-\|Q\| I$ is nonnegative and invertible, then for any unit vector $x \in H$, there exists constant $\delta>0$ such that $(X-\|Q\| I) x, x \geq \delta\|x\|^{2}$. Since $X=A^{*} X^{-t} A+Q$ and $Q \leq\|Q\| I$, we can conclude that

$$
\begin{aligned}
\delta\|x\|^{2} & \leq\langle(X-\|Q\| I) x, x\rangle \leq\langle(X-Q) x, x\rangle \\
& =\left(A^{*} X^{-t} A x, x\right)=\left\|X^{-(t / 2)} A x\right\|^{2} \leq\left\|X^{(t / 2)}\right\|^{2}\|A x\|^{2},
\end{aligned}
$$

that is, $\|A x\| \geq \sqrt{\delta}\left\|X^{-(t / 2)}\right\|^{-1}\|x\|$; this illustrates that $A$ is bounded below; it is a contradiction, so $\|X\|=\|Q\|$.

Theorem 5. If $X$ is a positive operator solution to equation (1), then

$$
\left(\lambda_{\max }(X)-\lambda_{\max }(Q)\right) \lambda_{\min }^{t}(X) \leq \lambda_{\max }\left(A^{*} A\right) \leq\left(\lambda_{\max }(X)-\lambda_{\min }(Q)\right) \lambda_{\max }^{t}(X)
$$

Proof. From $0<\lambda_{\min }(X) I \leq X \leq \lambda_{\max }(X) I$, we have $\mathrm{Q}+\left(A^{*} A / \lambda_{\max }^{t}(X)\right) \leq Q+A^{*} X^{-t} A \leq \mathrm{Q}+\left(A^{*} A / \lambda_{\min }^{t}(X)\right)$,

$$
\mathrm{Q}+\frac{A^{*} A}{\lambda_{\max }^{t}(X)} \leq X \leq \mathrm{Q}+\frac{A^{*} A}{\lambda_{\min }^{t}(X)} .
$$


From the first inequality of (10), we have $\left(A^{*} A / \lambda_{\max }^{t}(X)\right) \leq \lambda_{\max }(X-Q) \leq \lambda_{\max }(X)-\lambda_{\min }(Q)$, that is, $\lambda_{\text {max }}\left(A^{*} A\right) \leq\left(\lambda_{\text {max }}(X)-\lambda_{\text {min }}(Q)\right) \lambda_{\text {max }}^{t}(X)$.

From the second inequality of (10), we have $\lambda_{\max }(X) \leq \lambda_{\max }\left(Q+\left(A^{*} A / \lambda_{\min }^{t}(X)\right)\right) \leq \lambda_{\max }(Q)+\lambda_{\max }\left(A^{*}\right.$ $\left.A / \lambda_{\min }^{t}(X)\right)$, that is, $\lambda_{\max }\left(A^{*} A\right) \geq\left(\lambda_{\max }(X)-\lambda_{\max }(Q)\right)$ $\lambda_{\min }^{t}(X)$. Therefore, $\quad\left(\lambda_{\max }(X)-\lambda_{\max }(Q)\right) \lambda_{\min }{ }^{t}(X) \leq$ $\lambda_{\text {max }}\left(A^{*} A\right) \leq\left(\lambda_{\max }(X)-\lambda_{\text {min }}(Q)\right) \lambda_{\max }{ }^{t}(X)$.

Theorem 6. If $A$ is normal, $t=2^{m}$ and $A, Q, t$ satisfy $\left(t\|A\|^{2} a^{t-1} /\|Q\|^{2 t}\right)<1$, then equation (1) has positive operator solution, where $m$ is the positive integer and $a=\left\|Q+A^{*} Q^{-t} A\right\|$.

Proof. Consider the sequence of positive operators $\left\{X_{n}\right\}_{n=0}^{+\infty}$,

$$
X_{0}=Q, X_{k+1}=Q+A^{*} X_{k}^{-t} A, \quad k=0,1,2, \ldots
$$

According to the iteration sequence (11), $X_{i}$ is in the $C^{*}$-algebra generated by $A$ and $Q$. Because $A$ is normal, in accordance with Proposition 1 , for any $n=0,1,2, \ldots$, we have $A X_{n}=X_{n} A, X_{n+1} X_{n}=X_{n} X_{n+1}$. Since $X_{1}=Q+$ $A^{*} X_{0}^{-t} A \geq Q={ }_{X}^{0}$ and $X_{1}{ }^{-t} \leq X_{0}^{-t}$, it is easy to see $X_{2}=Q+A^{*} X_{1}^{-t} A \leq Q+A^{*} X_{0}^{-t} A={ }_{X}^{1}$; this implies $Q=X_{0} \leq$ $X_{2} \leq_{X}^{1}=Q+A^{*} Q_{-}^{t} A$. Successive analogy: we can prove

$$
Q=X_{0} \leq X_{2} \leq X_{4} \leq \ldots \leq X_{5} \leq X_{3} \leq X_{1}=Q+A^{*} Q^{-t} A,
$$

therefore the subsequence $\left\{X_{2 n}\right\}_{n=0}^{+\infty}$ and $\left\{X_{2 n+1}\right\}_{n=0}^{+\infty}$ both converge to positive operators. At the same time, for all nonnegative integers $i$, we have $Q \leq X_{i} \leq Q+A^{*} Q_{-}^{t} A$.

$\|Q\| \leq\|X\|_{i} \leq\left\|Q+A^{*} Q_{-}^{t} A\right\|$. Denote $\left\|Q+A^{*} Q_{-}^{t} A\right\|=a$, then

$$
\begin{aligned}
\left\|X_{2 k+1}-X_{2 k}\right\| & =\left\|A^{*} X_{2 k}^{-t} A-A^{*} X_{2 k-1}^{-t} A\right\| \leq\|A\|^{2}\left\|X_{2 k}^{-t}\left(X_{2 k-1}^{t}-X_{2 k}^{t}\right) X_{2 k-1}^{-t}\right\| \leq\|A\|^{2}\|Q\|^{-2 t}\left\|X_{2 k-1}^{t}-X_{2 k}^{t}\right\|, \\
\left\|X_{2 k-1}^{t}-X_{2 k}^{t}\right\| & =\left\|X_{2 k-1}^{(t / 2)}\left(X_{2 k-1}^{(t / 2)}-X_{2 k}^{(t / 2)}\right)+\left(X_{2 k-1}^{(t / 2)}-X_{2 k}^{(t / 2)}\right) X_{2 k}^{(t / 2)}\right\| \\
& \leq\left\|X_{2 k-1}^{(t / 2)}-X_{2 k}^{(t / 2)}\right\|\left(\left\|X_{2 k-1}^{(t / 2)}\right\|+\left\|X_{2 k}^{(t / 2)}\right\|\right) \leq 2 a^{(t / 2)}\left\|X_{2 k-1}^{(t / 2)}-X_{2 k}^{(t / 2)}\right\| .
\end{aligned}
$$

In the same way, we have

$$
\left\|X_{2 k-1}^{(t / 2)}-X_{2 k}^{(t / 2)}\right\| \leq 2 a^{(t / 4)}\left\|X_{2 k-1}^{(t / 4)}-X_{2 k}^{(t / 4)}\right\|, \ldots
$$

Successive analogy:

$$
\left\|X_{2 k-1}^{t}-X_{2 k}^{t}\right\| \leq\left(2 a^{(t / 2)}\right)\left(2 a^{(t / 4)}\right) \cdots\left(2 a^{\left(t / 2^{m}\right)}\right)\left\|X_{2 k-1}^{\left(t / 2^{m}\right)}-X_{2 k}^{\left(t / 2^{m}\right)}\right\|=2^{m} a^{t-1}\left\|X_{2 k-1}-X_{2 k}\right\| .
$$

Therefore, $\left\|X_{2 k+1}-X_{2 k}\right\| \leq t a^{t-1}\|A\|^{2}\|Q\|^{-2 t}\left\|X_{2 k-}^{1}-X_{2}^{k}\right\|$. Combined with the condition $\left(t\|A\|^{2} a^{t-1} /\|Q\|^{2 t}\right)<1$, we can know that subsequence $\left\{X_{2 n}\right\}_{n=0}^{+\infty}$ and $\left\{X_{2 n+1}\right\}_{n=0}^{+\infty}$ converge to the same positive operator, which is the positive operator solution to equation (1).

\section{Data Availability}

This paper is a theoretical analysis without data.

\section{Conflicts of Interest}

The author declares no conflicts of interest.

\section{Acknowledgments}

This work was supported by the National Natural Science Foundation of China (11301318) and the Natural Science Foundation of Shaanxi Educational Committee Grant (18JK0162). This work was also supported by the NSF of China.

\section{References}

[1] W. Pusz and S. L. Woronowicz, "Functional calculus for sesquilinear forms and the purification map," Reports on Mathematical Physics, vol. 8, no. 2, pp. 159-170, 1975.

[2] J. C. Engwerda, "On the existence of a positive definite solution of the matrix equation X+ATX-1A=I," Linear Algebra and Its Applications, vol. 194, pp. 91-108, 1993.

[3] W. L. Green and E. Kamen, "Stabilization of linear systems over a commutative normed algebra with applications to spatially distributed parameter dependent systems," SIAM Journal on Control and Optimization, vol. 23, pp. 1-18, 1985.

[4] R. S. Bucy, "A priori bounds for the riccati equation," in Contributions to Probability Theory, vol. 111, pp. 645-656, University of California Press, Berkeley, CA, USA, 1972.

[5] D. Yan and Z. Pan, "Positive solutions of nonlinear operator equations with sign-changing kernel and its applications," Applied Mathematics and Computation, vol. 230, no. 1, pp. 675-679, 2014.

[6] Y. Zhang, "On Hermitian positive definite solutions of matrix equation $\mathrm{X}+\mathrm{A} * \mathrm{X}-2 \mathrm{~A}=\mathrm{I}$," Linear Algebra and Its Applications, vol. 372, pp. 295-304, 2003. 
[7] K. F. Yang and H. K. Du, "Studies on the positive operator solutions to operator equations $X+A^{*} X^{-t} A=Q$," Acta Mathematica Scientia, vol. 26, no. 2, pp. 359-364, 2009, in Chinese.

[8] S. Esmaili and M. R. Eslahchi, "A modified spectral method for solving operator equations," Journal of Computational and Applied Mathematics, vol. 292, no. 15, pp. 105-135, 2016.

[9] C. Deng, "On the solutions of operator equation," Journal of Mathematical Analysis and Applications, vol. 398, no. 2, pp. 664-670, 2013.

[10] Y. Yang, "The iterative method for solving nonlinear matrix equation $X^{s}+A^{*} X^{-t} A=Q$," Applied Mathematics and Computation, vol. 188, no. 1, pp. 46-53, 2007.

[11] Y. Sang, "Multiple solutions for nonlinear operator equations under the condition of two pairs of paralleled lower and upper solutions," Computers \& Mathematics with Applications, vol. 63, no. 8, pp. 1349-1353, 2012.

[12] J. B. Conway, A Course in Operator Theory, American Mathematical Society Providence, Rhode Island, USA, 2000. 